\title{
RECENT DEVELOPMENTS IN INFINITE DIMENSIONAL HOLOMORPHY
}

\section{BY LEOPOLDO NACHBIN}

1. Introduction. There has been a recent and growing interest in holomorphy in infinite dimensional spaces; that is, the study of holomorphic mappings on open, or compact, or more general subsets of complex Banach spaces, or even of complex topological vector spaces which are locally convex, as well as of manifolds modeled after such spaces. This is an area of convergent attention of present day analysts and geometers.

Actually, such a field of research has old roots in the past. They stem from the investigation of holomorphic mappings in infinitely many variables and from the consideration of Taylor series-like expansions of functionals, discussed during the final quarter of the last century and the first quarter of this century. I refer you to the expositions in monograph form by Volterra, Pincherle, Paul Lévy and Hille-Phillips, for instance.

There is a huge literature on the subject covering the old-fashioned, the classical and the recent periods of the creation of this theory. An interesting historical survey has been made recently by Angus Taylor.

In this one-hour lecture, I shall try to describe some current progress, part of which is as yet unpublished. In so doing and by reason of limitation of time, I must be choosy. Needless to say, I will today confine myself to results which are closer to my heart.

2. Holomorphic mappings. Let me start with some notation and terminology.

$E$ and $F$ will denote complex topological vector spaces which are locally convex. During most of this lecture, you can think of $E$ and $F$ as just being in particular Banach spaces.

Passing from the finite dimensional situation to Banach spaces presents us with challenging problems. Passing from Banach spaces to locally convex spaces leads us to new challenges.

$U$ will denote a nonvoid open subset of $E$. $K$ will be a compact subset of E.

$\mathscr{L}_{s}\left({ }^{m} E ; F\right)$ will denote the vector space of all continuous symmetric $m$-linear mappings $A: E^{m} \rightarrow F$, where $E^{m}$ is the Cartesian $m$-power of $E$.

If $A \in \mathscr{L}_{s}\left({ }^{m} E ; F\right)$ and $x \in E$, I will write

An invited address delivered to the 77th Annual Meeting of the American Mathematical Society in Atlantic City, New Jersey, on January 22, 1971 ; partially supported by the National Science Foundation, Washington, D.C., and the Centro Brasileiro de Pesquisas Físicas, Rio de Janeiro, GB, Brasil; received by the editors November 21, 1972. 


$$
A \underbrace{(x, \ldots, x)}_{m}=A \cdot x^{m}
$$

for short. This notation makes formulas look the same way they do in one complex variable analysis, which has psychological advantages.

$\mathscr{P}\left({ }^{m} E ; F\right)$ will denote the vector space of all continuous $m$-homogeneous polynomials $P: E \rightarrow F$.

To every $A \in \mathscr{L}_{s}\left({ }^{m} E ; F\right)$ there corresponds $P=\hat{A} \in \mathscr{P}\left({ }^{m} E ; F\right)$ given by $P(x)=A \cdot x^{m}$ for every $x \in E$. The mapping

$$
A \in \mathscr{L}_{s}\left({ }^{m} E ; F\right) \mapsto P=\hat{A} \in \mathscr{P}\left({ }^{m} E ; F\right)
$$

is linear and bijective.

If $E$ and $F$ are Banach spaces, then $\mathscr{L}_{s}\left({ }^{m} E ; F\right)$ and $\mathscr{P}\left({ }^{m} E ; F\right)$ are naturally Banach spaces which are homeomorphic but not necessarily isometric under the aforementioned mapping.

In all this, we have $m=0,1, \ldots$ with the usual interpretation for $m=0$.

A mapping $f: U \rightarrow F$ is said to be holomorphic on $U$ if, for every $\xi \in U$, there is a sequence of coefficients $A_{m} \in \mathscr{L}_{s}\left({ }^{m} E ; F\right), m=0,1, \ldots$, such that, for every continuous seminorm $\beta$ on $F$, we can find an open subset $V$, $\xi \in V \subset U$, such that

$$
\lim _{M \rightarrow+\infty} \beta\left[f(x)-\sum_{m=0}^{M} A_{m} \cdot(x-\xi)^{m}\right]=0
$$

uniformly for $x \in V$.

If $F$ is a Hausdorff space, for each given $f$ and $\xi$, the sequence $\left(A_{m}\right)$ is unique. I will then write

$$
d^{m} f(\xi)=m ! A_{m} \in \mathscr{L}_{s}\left({ }^{m} E ; F\right), \quad \hat{d}^{m} f(\xi)=m ! \hat{A}_{m} \in \mathscr{P}\left({ }^{m} E ; F\right),
$$

for the $m$ th differentials of $f$ at $\xi$ viewed as a continuous symmetric $m$-linear mapping and as a continuous $m$-homogeneous polynomial, respectively.

I shall denote by $\mathscr{H}(U ; F)$ the vector space of all holomorphic mappings $f: U \rightarrow F$.

For the sake of simplicity, let us assume from now on that $E$ and $F$ are Banach spaces, unless the contrary is explicitly stated.

3. Domains of holomorphy. The classical Cartan-Thullen theorem about domains of holomorphy in finite dimensions suggests the following definition.

A subset $X$ of $U$ is said to be $\mathscr{H}(U ; C)$-bounding if every $f \in \mathscr{H}(U ; \boldsymbol{C})$ is bounded on $X$. 
THEOREM 1. Assume that from every bounded sequence in the dual space $E^{\prime}$, it is possible to extract a weak-star convergent subsequence, in particular that $E$ is separable or reflexive. Then every closed $\mathscr{H}(E ; C)$-bounding subset must be compact.

This result was noticed for $E$ a Hilbert space independently by Alexander and Dineen. In the preceding general form, it is due independently to Dineen and Hirschowitz.

There is an example due to Dineen of a closed $\mathscr{H}(E ; C)$-bounding subset which is not compact, when $E=l^{\infty}$, the Banach space of all bounded sequences of complex numbers.

A subset $X$ of $U$ is said to be $U$-bounded if $X$ is bounded in $E$ and $X$ is bounded away from the boundary of $U$, that is $\operatorname{dist}(X, \partial U)>0$.

The mapping $f \in \mathscr{H}(U ; F)$ is said to be of bounded type if $f$ is bounded on every $U$-bounded subset.

Let $\mathscr{H}_{b}(U ; F)$ be the vector subspace of $\mathscr{H}(U ; F)$ of all such $f$ of bounded type.

On $\mathscr{H}_{b}(U ; F)$ we use the topology $\mathscr{T}_{b}$ of uniform convergence on the $U$-bounded subsets, which is given by the family of seminorms

$$
f \in \mathscr{H}_{b}(U ; F) \mapsto \sup _{x \in X}\|f(x)\| \in \boldsymbol{R}_{+},
$$

where $X$ is $U$-bounded. Then $\mathscr{H}_{b}(U ; F)$ is a Fréchet space, that is, a complete metrizable locally convex space.

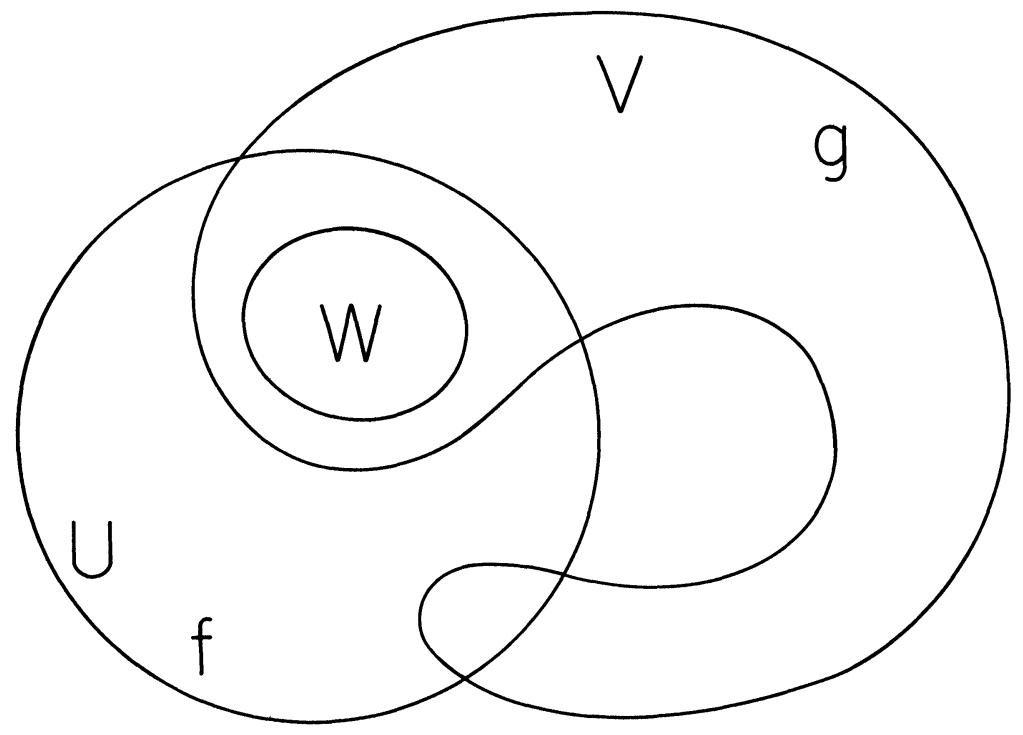

FIGURE 1 
It is clear that $\mathscr{H}_{b}(U ; F)=\mathscr{H}(U ; F)$ if $E$ is finite dimensional or in the trivial case $F=0$. It is conjectured that these spaces are different if $E$ is infinite dimensional and $F \neq 0$. As noticed by Dineen, these spaces are indeed different if $F \neq 0$ and if there is a sequence in $E^{\prime}$ which is weakstar convergent but not norm convergent. Actually it is conjectured that every infinite dimensional space $E$ satisfies this last stated condition.

$U$ is said to be an $\mathscr{H}_{b}$-domain of holomorphy if $U$ is connected and it is impossible to find open, connected and nonvoid subsets $V, W$ in $E, W \subset$ $U \cap V, V \notin U$, such that, for every $f \in \mathscr{H}_{b}(U ; C)$, the restriction of $f$ to $W$ has a continuation to some $g \in \mathscr{H}_{b}(V ; \boldsymbol{C})$. (See Figure 1.)

$U$ is said to be an $\mathscr{H}_{b}$-domain of existence if $U$ is connected and there is $f \in \mathscr{H}_{b}(U ; C)$ having $U$ as its natural domain of $\mathscr{H}_{b}$-existence, that is, $f$ is everywhere $\mathscr{H}_{b}$-singular on the boundary of $U$. This means that, for every open, connected and nonvoid subsets $V, W$ in $E, W \subset U \cap V, V \notin U$, the restriction of $f$ to $W$ has no continuation to some $g \in \mathscr{H}_{b}(V ; C)$. (See Figure 1.)

$U$ is said to be $\mathscr{H}_{b}$-holomorphically convex if, for every $U$-bounded set $X$, the hull of $X$ with respect to $\mathscr{H}_{b}(U ; C), \hat{X}_{\mathscr{H}_{b}(U ; \boldsymbol{C})}$, is also $U$-bounded; this hull is defined as the set of all $t \in U$ such that the maximum modulus style estimate

$$
|f(t)| \leqq \sup _{x \in X}|f(x)|
$$

holds for every $f \in \mathscr{H}_{b}(U ; \boldsymbol{C})$.

A subset $X$ of $U$ is said to be $\mathscr{H}_{b}(U ; \boldsymbol{C})$-bounding if every $f \in \mathscr{H}_{b}(U ; \boldsymbol{C})$ is bounded on $X$. This is analogous to a previous definition we gave with $\mathscr{H}_{b}(U ; \boldsymbol{C})$ now in place of $\mathscr{H}(U ; \boldsymbol{C})$.

We then have the following theorem of the Cartan-Thullen style, which was noticed independently by Dineen and Matos.

THEOREM 2. Let $U$ be connected. The following conditions are equivalent:

(1) $U$ is an $\mathscr{H}_{b}$-domain of holomorphy;

(2) $U$ is $\mathscr{H}_{b}$-holomorphically convex;

(3) a subset $X$ of $U$ is $U$-bounded if and only if $X$ is $\mathscr{H}_{b}(U ; C)$-bounding. Moreover, if $E$ is separable, the above are also equivalent to

(4) $U$ is an $\mathscr{H}_{b}$-domain of existence;

(5) the complement in $\mathscr{H}_{b}(U ; \boldsymbol{C})$ of the set of all $f \in \mathscr{H}_{b}(U ; C)$ having $U$ as its natural $\mathscr{H}_{b}$-domain of existence is meager, that is, of Baire first category.

Hirschowitz has found an example showing that the hypothesis of separability for $E$ cannot be dropped in the above theorem. In his example, $U$ is an open ball in the Banach space $E=\mathscr{C}(X ; C)$ of all continuous complex-valued functions on the compact space $X=[0, \Omega]$ of the cardinal 
numbers between 0 and $\Omega$, the first uncountable cardinal.

The full analogue of the Cartan-Thullen theorem with the space $\mathscr{H}(U ; C)$ in place of $\mathscr{H}_{b}(U ; C)$ is still an open question which is being investigated by various mathematicians.

This has to do with characterization of domains of holomorphy, domains of existence, holomorphic convexity, pseudoconvex domains, holomorphic continuation and envelopes of holomorphy as Riemann domains or manifolds spread over Banach or locally convex spaces, plurisubharmonic functions, Runge sets, etc., in infinite dimensions.

Several concepts and conditions which coincide in the finite dimensional case fail to do so in infinite dimensions.

There are plenty of closely related candidates for the wanted results.

We have to distinguish carefully between the collection of all bounded subsets, and the collection of all open covers, as well as between holomorphic functions and holomorphic mappings.

A description of these developments goes beyond the limits of this onehour lecture.

Recent work on this type, and other aspects, of infinite dimensional complex analysis has been carried on mostly by Alexander, Bremermann, Coeuré, Dineen, Earle, Greenfield, Harris, Hirschowitz, Lelong, Matos, Noverraz, Rickart, Schottenloher and Wallach.

4. Topological properties. The question arises of what topology to use on $\mathscr{H}(U ; F)$.

In the finite dimensional situation, there is the natural candidate, the compact-open topology $\mathscr{T}_{0}$ of order zero, which coincides with the compact-open topology $\mathscr{T}_{\infty}$ of infinite order.

In the infinite dimensional situation, there are still the compact-open topology $\mathscr{T}_{0}$ of order zero defined by the family of seminorms

$$
f \in \mathscr{H}(U ; F) \mapsto \sup _{x \in \mathbf{K}}\|f(x)\| \in \boldsymbol{R}_{+},
$$

where $K \subset U$ is compact, as well as the compact-open topology $\mathscr{T}_{\infty}$ of infinite order, defined by the family of seminorms

$$
f \in \mathscr{H}(U ; F) \mapsto \sup _{x \in \mathbf{K}}\left\|d^{m} f(x)\right\| \in \boldsymbol{R}_{+},
$$

where $K \subset U$ is compact and $m=0,1, \ldots$. These two topologies may be different and in several respects they lack the right properties which they have in the finite dimensional case. This calls for further investigation.

A seminorm $p$ on $\mathscr{H}(U ; F)$ is said to be ported by a compact subset $K$ of $U$ if, for every open subset $V$, where $K \subset V \subset U$, there is a real number $c(V)>0$ such that we have the estimate 


$$
p(f) \leqq c(V) \sup _{x \in V}\|f(x)\|
$$

for all $f \in \mathscr{H}(U ; F)$. The simple intuitive idea behind this definition is that, if a variable mapping $f \in \mathscr{H}(U ; F)$ tends to 0 uniformly on some neighborhood of $K$, then $p(f)$ should tend to 0 .

The topology $\mathscr{T}_{\omega}$ on $\mathscr{H}(U ; F)$ is defined by all seminorms on $\mathscr{H}(U ; F)$ each of which is ported by some compact subset of $U$.

We have $\mathscr{T}_{0}=\mathscr{T}_{\infty}=\mathscr{T}_{\omega}$ if $\operatorname{dim} E<\infty$ or in the trivial case $F=0$, whereas $\mathscr{T}_{0}<\mathscr{T}_{\infty}<\mathscr{T}_{\omega}$ if $\operatorname{dim} E=\infty$ and $F \neq 0$.

The three topologies $\mathscr{T}_{0}, \mathscr{T}_{\infty}$ and $\mathscr{T}_{\omega}$ define the same notion of bounded subset of $\mathscr{H}(U ; F)$.

The following nice and basic result is due to Dineen.

THEOREM 3. For "many" separable Banach spaces $E$, if $U$ is balanced with respect to some point, then $\mathscr{T}_{\omega}$ is the largest locally convex topology on $\mathscr{H}(U ; C)$ having the same collection of bounded subsets as $\mathscr{T}_{0}$. Hence $\mathscr{T}_{\omega}$ is bornological.

In the above theorem, it is assumed that the space $E$ has a basis $\left(u_{n}\right)$, $n=0,1, \ldots$, such that, for every

$$
\alpha=\left(\alpha_{0}, \ldots, \alpha_{n}, \ldots\right) \in c_{0},
$$

the Banach space of all sequences of complex numbers tending to zero, and for every

$$
x=\sum_{n=0}^{+\infty} \beta_{n} u_{n} \in E
$$

it results that

$$
\alpha \cdot x=\sum_{n=0}^{+\infty} \alpha_{n} \beta_{n} u_{n}
$$

converges in $E$ and the bilinear mapping

$$
(\alpha, x) \in c_{0} \times E \mapsto \alpha \cdot x \in E
$$

is continuous.

It is conjectured that the preceding theorem holds for every separable Banach space $E$ and every $U$. Dineen showed that this theorem breaks down for $E=l^{\infty}$.

On the other hand, Coeure subsequently found another instance in which the conclusion of the above theorem is true.

If $K \subset E$ is compact, we have the vector space $\mathscr{H}(K ; F)$ of all germs of holomorphic mappings around $K$ into $F$.

Consider the big set 


$$
\bigcup_{U \supset \boldsymbol{K}} \mathscr{H}(U ; F)
$$

where the union is taken over all $U$ containing $K$. An equivalence relation on this big set is defined by saying that $f$ and $g$ in that big set are equivalent modulo $K$ when $f$ and $g$ coincide in a neighborhood of $K$. The quotient of that big set by such an equivalence relation is the set $\mathscr{H}(K ; F)$.

We have the natural mapping $\mathscr{H}(U ; F) \rightarrow \mathscr{H}(K ; F)$ if $U \supset K$, coming from the quotient mapping. There is one and only one way of making $\mathscr{H}(K ; F)$ into a vector space so that this natural mapping is linear for every $U \supset K$.

The natural topology $\mathscr{T}_{\omega}$ on $\mathscr{H}(K ; F)$ is obtained by considering $\mathscr{H}(K ; F)$ as a locally convex inductive (or direct) limit.

$$
\mathscr{H}(K ; F)=\underset{U \supset \boldsymbol{K}}{\lim } \mathscr{H}_{b}(U ; F)=\underset{U \supset \boldsymbol{K}}{\lim } \mathscr{H}(U ; F)
$$

where $\mathscr{H}_{b}(U ; F)$ has its topology $\mathscr{T}_{b}$ and $\mathscr{H}(U ; F)$ has its topology $\mathscr{T}_{\omega}$. Both inductive limits give the same topology $\mathscr{T}_{\omega}$ on $\mathscr{H}(K ; F)$.

The question arises of finding out whether, conversely, we have the topological projective (or inverse) limit relation

$$
\mathscr{H}(U ; F)=\lim _{\overleftarrow{K \subset U}} \mathscr{H}(K ; F),
$$

where $\mathscr{H}(U ; F)$ is endowed with its topology $\mathscr{T}_{\omega}$ and $\mathscr{H}(K ; F)$ is endowed with the topology $\mathscr{T}_{\omega}$.

To answer this question, we define the compact subset $K$ to be $U$-Runge if $K \subset U$ and the image of $\mathscr{H}(U ; C)$ in $\mathscr{H}(K ; C)$ by the natural mapping $\mathscr{H}(U ; C) \rightarrow \mathscr{H}(K ; C)$ is $\mathscr{T}_{\omega}$-dense in $\mathscr{H}(K ; C)$.

Then, if every compact subset of $U$ is contained in some $U$-Runge compact subset of $U$, the preceding topological projective limit relation holds, as shown by Chae.

Actually, it is conjectured that every compact subset of $U$ is contained in some $U$-Runge compact subset; to my knowledge, however, this statement remains unsettled even in the finite dimensional case. This conjecture may be phrased in more general partial differential operator terms.

It is curious to realize that the topological study of $\mathscr{H}(K ; C)$ was considered as being more sophisticated than that of $\mathscr{H}(U ; C)$ for a finite dimensional space $E$. It was only after a classical article by Dieudonné and Schwartz on inductive limits that $\mathscr{H}(K ; \boldsymbol{C})$ could be treated as a locally convex space. However, when we jump to an infinite dimensional space $E$, it is the topological study of $\mathscr{H}(U ; C)$ which becomes more sophisticated than that of $\mathscr{H}(K ; C)$. 
A lot of work on the topological aspects of spaces of holomorphic mappings in infinite dimensions has been done by Alexander, Aron, Barroso, Chae, Coeuré, Dineen, Hirschowitz, Matos and Pisanelli.

5. Convolution operators. We now turn to convolution operators.

Let $\mathscr{P}_{N}\left({ }^{m} E ; \boldsymbol{C}\right)$ be the Banach space of all nuclear, complex-valued, $m$-homogeneous polynomials on $E$, as defined by Grothendieck.

An entire function $f \in \mathscr{H}(E ; C)$ is said to be nuclearly entire of bounded type if

$$
\hat{d}^{m} f(0) \in \mathscr{P}_{N}\left({ }^{m} E ; C\right)
$$

for $m=0,1, \ldots$, and

$$
\left(\left.\left\|\frac{1}{m !} \hat{d}^{m} f(0)\right\|\right|_{N} ^{1 / m} \rightarrow 0\right.
$$

as $m \rightarrow+\infty$, where the index $N$ denotes the nuclear norm on the space $\mathscr{P}_{N}\left({ }^{m} E ; C\right)$.

We represent by $\mathscr{H}_{N b}(E ; C)$ the vector subspace of $\mathscr{H}(E ; C)$ of all such $f$.

We shall use on $\mathscr{H}_{N b}(E ; C)$ the topology $\mathscr{T}_{N b}$ defined by the family of seminorms

$$
f \mapsto \sum_{m=0}^{+\infty} r^{m} \cdot\left\|\frac{1}{m !} \hat{d}^{m} f(0)\right\|_{N}
$$

for every real number $r \geqq 0$. This topology $\mathscr{T}_{N b}$ is locally convex, metrizable and complete; that is $\mathscr{H}_{N b}(E ; C)$ is a Fréchet space.

A convolution operator $\mathcal{O}$ on $\mathscr{H}_{N b}(E ; C)$ is a continuous linear mapping

$$
\mathcal{O}: \mathscr{H}_{N b}(E ; \boldsymbol{C}) \rightarrow \mathscr{H}_{N b}(E ; \boldsymbol{C})
$$

which commutes with translations by elements of $E$. Such a convolution operator in this case is actually a constant coefficient, linear, differential operator of finite or infinite order.

A nuclear exponential-polynomial is an element of $\mathscr{H}_{N b}(E ; C)$ of the form $p \exp (\varphi)$, where $p$ is a nuclear complex-valued polynomial on $E$ and $\varphi \in E^{\prime}$ is a continuous linear form on $E$.

The following approximation and existence theorem of the Malgrange type was proved by Gupta.

THEOREM 4. If $\mathcal{O}$ is a convolution operator on $\mathscr{H}_{N b}(E ; C)$, then the vector subspace $\mathcal{O}^{-1}(0)$ where $\mathcal{O}$ vanishes is the closure of its vector subspace formed by the finite sums of the nuclear exponential-polynomials belonging to $\mathcal{O}^{-1}(0)$. Moreover 


$$
\mathcal{O}\left[\mathscr{H}_{N b}(E ; \boldsymbol{C})\right]=\mathscr{H}_{N b}(E ; \boldsymbol{C})
$$

provided $\mathcal{O} \neq 0$.

An extension of this result to the space of nuclearly entire functions $\mathscr{H}_{N}(E ; C)$ not necessarily of bounded type has been made.

The bounded nuclear case has been extended by Matos to a locally convex space $E$ whose strong dual space $E^{\prime}$ is metrizable.

Dwyer has done work along this line when $E$ is a Hilbert space and we consider entire functions of the Hilbert-Schmidt type.

Boland has been investigating this kind of a result by using weights on the Banach space $E$.

The cases of the current type $\mathscr{H}_{b}(E ; C)$ and $\mathscr{H}(E ; C)$ remain untouched and at present seem hard.

It was the success of these studies in the nuclear type cases $\mathscr{H}_{N b}(E ; C)$ and $\mathscr{H}_{N}(E ; C)$, and the existence of the current type, that prompted the introduction of the concept of holomorphy type from $E$ to $F$, such as the current type, the nuclear type, the Hilbert-Schmidt type, etc.

We notice that theorems of the Cartan-Thullen style, or of the Malgrange style, in infinite dimensions are harder for the current type, but become more accessible for the bounded case, or the bounded nuclear case.

6. Uniform holomorphy. Let us now give up the restriction that $E$ and $F$ are Banach spaces and assume that they are locally convex spaces.

I shall state next a result which is typical of this more general situation and does not have significance in the normed case.

To start with, we set the following simple notation. If $\alpha$ is a seminorm on $E$, I denote by $E_{\alpha}$ the vector space $E$ seminormed by $\alpha$, and represent by $E / \alpha$ the quotient normed space $E_{\alpha} / \alpha^{-1}(0)$ of $E_{\alpha}$ by the vector subspace $\alpha^{-1}(0)$ where $\alpha$ vanishes. The same is done for the space $F$.

First I shall assume that the space $F$ is seminormed. Then I say that the mapping $f \in \mathscr{H}(U ; F)$ is uniformly holomorphic if there is a cover $\mathscr{C}$ of $U$ by nonvoid open subsets of $U$ and if there is a continuous seminorm $\alpha$ on $E$ such that, for every $W \in \mathscr{C}$, there is $V \subset E$ which is open with respect to $\alpha$, where $W \subset V$, and the restriction of $f$ to $W$ has an extension $g$ to $V$ which is holomorphic with respect to $\alpha$ on $V$ with values in $F$. (See Figure 1.)

More generally, when the space $F$ is allowed to be locally convex, I say that the mapping $f \in \mathscr{H}(U ; F)$ is uniformly holomorphic if the mapping $f \in \mathscr{H}\left(U ; F_{\beta}\right)$ is uniformly holomorphic in the above sense for every continuous seminorm $\beta$ on $F$.

THEOREM 5. Assume that the space $E$ satisfies the following condition: The set of seminorms $\alpha$ on $E$, rendering the quotient mapping $E \rightarrow E / \alpha$ continuous and open, is directed and defines the topology E. Then every 
mapping $f \in \mathscr{H}(U ; F)$ is uniformly holomorphic.

When $F$ is seminormed, this result can be reformulated as meaning that every mapping $f \in \mathscr{H}(U ; F)$ is globally holomorphic with respect to some continuous seminorm on $E$; but then we must use Riemann domains as manifolds spread over normed spaces, in order to avoid multivalent holomorphic mappings.

The preceding theorem is actually a generalization of the following classical remark: if $I$ is an infinite set, then every holomorphic function $f \in \mathscr{H}\left(\boldsymbol{C}^{I} ; \boldsymbol{C}\right)$ of infinitely many variables actually depends only on finitely many such variables.

In the case of a locally convex space $E$, use of uniform holomorphy has been made by Dineen and Noverraz to discuss mutual implications of various forms of conditions for $U$ to be a domain of holomorphy, or a domain of existence, or holomorphically convex, or plurisubharmonically convex, or pseudoconvex, or polynomially convex.

7. Concluding remarks. Well, I hope to have succeeded in giving all of you an impression of a variety of directions of the current efforts to push ahead investigations on infinite dimensional holomorphy.

There are also other tendencies that I was not able to touch upon, such as the study of infinite dimensional analytic geometry carried on by Douady, Ramis and Ruget; or of infinite dimensional analytic manifolds; or applications of complex analysis in infinite dimensions to physics; etc.

In all these areas, the problems outnumber the solutions.

\section{BIBLIOGRAPHY \\ (References to recent work reflecting some of the trends.)}

1. H. Alexander, Analytic functions on Banach spaces, Thesis, University of California, Berkeley, Calif., 1968.

2. R. M. Aron, Sur la topologie bornologique pour l'espace d'applications holomorphes, C. R. Acad. Sci. Paris Sér. A-B 272 (1971), A872-A873. MR 43 \# 5295.

3. Holomorphic functions on balanced subsets of a Banach space, Bull. Amer. Math. Soc. 78 (1972), 624-627.

4. - Holomorphy types for open subsets of a Banach space, Studia Math. 45 (to appear).

5. The bornological topology on the space of holomorphic mappings on a Banach space, Math. Ann. (to appear).

6. R. M. Aron and J. A. Cima, A theorem on holomorphic mappings into Banach spaces with basis, Proc. Amer. Math. Soc. 36 (1972), 289-292.

7. R. R. Baldino, Aplicações holomorfas em produtos cartesianos, Tese, Instituto de Matemática Pura e Aplicada, Rio de Janeiro, Brasil, 1972.

8. J. A. Barroso, Topologies sur les espaces d'applications holomorphes entre des espaces localement convexes, C. R. Acad. Sci. Paris Sér. A-B 271 (1970), A264-A265. MR 42 \# 6594.

9. —— Topologias nos espaços de aplicações holomorfas entre espaços localmente convexos, An. Acad. Brasil. Ci. 43 (1971), 527-546.

10. J. A. Barroso et L. Nachbin, Sur certaines propriétés bornologiques des espaces d'applications holomorphes, Troisième Colloq. sur l'Analyse Fonctionnelle (Liège, 1970), Centre Belge de Recherches Mathématiques, Vander, Belgium, 1971, pp. 47-55.

11. S. Baryton, Fonctions A-analytiques, C. R. Acad. Sci. Paris Sér. A-B 270 (1970), 
A1586-A1588. MR 42 \#8277.

12. J. Bochnak, Analytic functions in Banach spaces, Studia Math. 35 (1970), 273-292. MR 42 \# 8275.

13. J. Bochnak et J. Siciak, Fonctions analytiques dans les espaces vectoriels topologiques réels au complexes, C. R. Acad. Sci. Paris Sér. A-B 270 (1970), A643-A646. MR 43 \# 7920.

14. - Remarks on domains of holomorphy in topological vector spaces, Bull. Acad. Polon. Sci. 19 (1971), 9-12.

15. Polynomials and multilinear mappings in topological vector spaces, Studia Math. 39 (1971), 59-76.

16. - Analytic functions in topological vector spaces, Studia Math. 39 (1971), $77-112$.

17. P. J. Boland, Espaces pondérés de fonctions entières et de fonctions entières nucléaires sur un espace de Banach, C. R. Acad. Sci. Paris Sér. A-B 275 (1972), A587-A590.

18. - Some spaces of entire and nuclearly entire functions on a Banach space, J. Reine Angew. Math. (to appear in two parts).

19. N. Bourbaki, Éléments de mathématique. Fasc. XXXIII; XXXVI. Variétés différentielles et analytiques. Fascicule de résultats (Para. 1-7; 8-15), Actualités Sci. Indust., nos. 1333, 1347, Hermann, Paris, 1967, 1971. MR 36 \# 2161; MR 43 \# 6834.

20. H. J. Bremermann, Holomorphic functionals and complex convexity in Banach spaces, Pacific J. Math. 7 (1957), 811-831. MR 19, 567.

21. The envelopes of holomorphy of tube domains in infinite dimensional Banach spaces, Pacific J. Math. 10 (1960), 1149-1153. MR 22 \#9882.

22. - Pseudo-convex domains in linear topological spaces, Proc. Conference on Complex Analysis (Minneapolis, 1964), Springer-Verlag, Berlin, 1965, pp. 182-186. MR 31 \# 583.

23. H. Cartan, Thèse de Douady, Séminaire Bourbaki 1965/66, Exposé 296 (01-16), Benjamin, New York, 1966.

24. - Sous-ensembles analytiques d'une variété banachique complexe (d'après J.-P. Ramis), Séminaire Bourbaki 1968/69, Lecture Notes in Math., vol. 179, SpringerVerlag, Berlin, 1971, pp. 123-138.

25. - Sur l'anneau des germes de fonctions holomorphes dans un espace de Banach, Espaces Analytiques (Séminaires Bucharest, 1969), Editura Acad. R.S.R., Bucharest, 1971, pp. 129-135. MR 43 \# 6460.

26. S. B. Chae, Sur les espaces localement convexes de germes holomorphes, C. R. Acad. Sci. Paris Sér. A-B 271 (1970), 990-991.

27. — Holomorphic germs on Banach spaces, Ann. Inst. Fourier (Grenoble) 21 (1971), 107-141.

28. J. A. Cima. See Aron, R. M. [6].

29. G. Coeuré, Le théorème de convergence dans les espaces localement convexes complexes, C. R. Acad. Sci. Paris Sér. A-B 264 (1967), A287-A290. MR 38 \# 338.

30. - Fonctions plurisousharmoniques et fonctions $C$-analytiques à une infinité de variables, C. R. Acad. Sci. Paris Sér. A-B 267 (1968), A440-A442; A473-A476 and 816. MR 38 \#4708.

31. - Prolongement analytique, Séminaire Pierre Lelong 1969, Lecture Notes in Math., vol. 116, Springer-Verlag, Berlin, 1970, pp. 128-132.

32. - Fonctions plurisousharmoniques sur les espaces vectoriels topologiques et applications à l'étude des fonctions analytiques, Ann. Inst. Fourier (Grenoble) 20 (1970), 361-432. MR 43 \# 564.

33. - Fonctionnelles analytiques sur certains espaces de Banach, Ann. Inst. Fourier (Grenoble) 21 (1971), 15-21.

34. - Prolongement analytique en dimension infinie, Sympos. Analysis (Recife, 1972), Lecture Notes in Math., Springer-Verlag, Berlin (to appear).

35. - Espaces normés $\mathcal{O}$-complets, Colloq. Analyse (Rio de Janeiro, 1972), Hermann, Paris (to appear).

36. J.-F. Colombeau, Quelques exemples singuliers d'applications G-analytiques, analytiques et différentiables en dimension infinie, C. R. Acad. Sci. Paris Sér. A-B 273 (1971), A158-A160. MR 44 \# 765.

37. J.-F. Colombeau et D. Lazet, Sur les théorèmes de Vitali et de Montel en dimension infinie, C. R. Acad. Sci. Paris Sér. A-B 274 (1972), A185-A187.

38. J.-F. Colombeau, Sur les applications G-analytiques et analytiques en dimension infinie, Séminaire Pierre Lelong 1971/72, Lecture Notes in Math., Springer-Verlag, Berlin (to appear).

39. S. Dineen, Topologie de Nachbin et prolongement analytique en dimension infinie, 
C. R. Acad. Sci. Paris Sér. A-B 271 (1970), A643-A644. MR. 43 \# 887.

40. - Holomorphic functions on a Banach space, Bull. Amer. Math. Soc. 76 (1970), 883-886. MR 41 \# 4216.

41. - The Cartan-Thullen theorem for Banach spaces, Ann. Scuola Norm. Sup. Pisa (3) 24 (1970), 667-676. MR 43 \# 3487.

42. - Bounding subsets of a Banach space, Math. Ann. 192 (1971), 61-70.

43. - Runge domains in Banach spaces, Proc. Roy. Irish Acad. 71 (1971), 85-89.

44. S. Dineen et A. Hirschowitz, Sur le théorème de Levi banachique, C. R. Acad. Sci.

Paris Sér. A-B 272 (1971), A1245-A1247. MR 43 \# 3488.

45. - Holomorphy types on a Banach space, Studia Math. 39 (1971), 241-288.

46. Unbounded holomorphic functions on a Banach space, J. London Math. Soc.

4 (1972), 461-465.

47. - Holomorphic functions on $\left(c_{0}, X_{b}\right)$-modules, Math. Ann. 196 (1972), 106-116.

48. - Fonctions analytiques dans les espaces vectoriels topologiques localement

convexes, C. R. Acad. Sci. Paris Sér. A-B 274 (1972), A544-A546.

49. Convexité holomorphe en dimension infinie, Séminaire Pierre Lelong 1970/71,

Lecture Notes in Math., vol. 275, Springer-Verlag, Berlin, 1972, pp. 177-181.

50. - Holomorphic functions on locally convex topological vector spaces, Ann.

Inst. Fourier (Grenoble) (to appear in two parts).

51. - Holomorphically significant properties of topological vector spaces, Colloq.

sur les Fonctions Analytiques de Plusieurs Variables Complexes (Paris, 1972), Centre

National de la Recherche Scientifique, Paris (to appear).

52. S. Dineen and L. Nachbin, Entire functions of exponential type bounded on the real

axis and Fourier transforms of distributions with bounded supports, Israel J. Math. (to appear).

53. A. Douady, Le problème des modules pour les sous-espaces analytiques compacts

d'un espace analytique donné, Ann. Inst. Fourier (Grenoble) 16 (1966), 1-95. MR 34 \# 2940.

54. T. A. W. Dwyer, Partial differential equations in Fisher-Fock spaces for the Hilbert-

Schmidt holomorphy type, Bull. Amer. Math. Soc. 77 (1971), 725-730.

55. ㄱ. Partial differential equations in holomorphic Fock spaces, Sympos. Analysis

(Recife, 1972), Lecture Notes in Math., Springer-Verlag, Berlin (to appear).

56. - Holomorphic representation of tempered distributions and weighted Fock spaces, Colloq. Analyse (Rio de Janeiro, 1972), Hermann, Paris (to appear).

57. C. J. Earle and R. S. Hamilton, $A$ fixed point theorem for holomorphic mappings,

Proc. Sympos. Pure Math., vol. 16, Amer. Math. Soc., Providence, R.I., 1970, pp. 61-65.

MR 42 \#918.

58. B. W. Glickfeld, The Riemann sphere of a commutative Banach algebra, Trans.

Amer. Math. Soc. 134 (1968), 1-28. MR 37 \# 3360.

59. The theory of analytic functions in commutative Banach algebras with involu-

tion, Ann. Mat. Pura Appl. (4) 86 (1970), 61-77. MR 42 \# 6625.

60. - Meromorphic functions of elements of a commutative Banach algebra, Trans.

Amer. Math. Soc. 151 (1970), 293-307. MR 41 \# 8993.

61. - On the inverse function theorem in commutative Banach algebras, Illinois $\mathrm{J}$.

Math. 15 (1971), 212-221. MR 42 \# 8287.

62. S. J. Greenfield and N. R. Wallach, The Hilbert ball and bi-ball are holomorphically inequivalent, Bull. Amer. Math. Soc. 77 (1971), 261-263. MR 42 \# 3815.

63. - Automorphism groups of bounded domains in Banach spaces, Trans. Amer.

Math. Soc. 166 (1972), 45-57.

64. L. Gruman et C. O. Kiselman, Le problème de Levi dans les espaces de Banach à base,

C. R. Acad. Sci. Paris Sér. A-B 274 (1972), A1296-A1299.

65. L. Gruman, The Levi problem in certain infinite dimensional vector spaces, Illinois J.

Math. (to appear).

66. Le problème de Levi en dimension infinie, Colloq. sur les Fonctions Analytiques de Plusieurs Variables Complexes (Paris, 1972), Centre National de la Recherche Scientifique, Paris (to appear).

67. C. P. Gupta, Malgrange theorem for nuclearly entire functions of bounded type on a

Banach space, Notas de Matemática, no. 37, Instituto de Matemática Pura e Aplicada, Rio de Janeiro, Brasil, 1968.

68. - Convolution operators and holomorphic mappings on a Banach space, Séminaire d'Analyse Moderne, 2, Université de Sherbrooke, Canada, 1969.

69. - On the Malgrange theorem for nuclearly entire functions of bounded type on a Banach space, Nederl. Akad. Wetensch. Proc. Ser. A $73=$ Indag. Math. 32 (1970), 356-358.

70. R. S. Hamilton. See Earle, C. J. [57].

71. L. A. Harris, Schwarz's lemma in normed linear spaces, Proc. Nat. Acad. Sci. U.S.A. 
62 (1969), 1014-1017. MR 43 \#936.

72. The numerical range of holomorphic functions in Banach spaces, Amer. J. Math. 93 (1971), 1005-1019.

73., A continuous form of Schwarz's lemma in normed linear spaces, Pacific J. Math. 38 (1971), 635-639.

74. Bounds on the derivatives of holomorphic functions of vectors, Colloq. Analyse (Rio de Janeiro, 1972), Hermann, Paris (to appear).

75. T. L. Hayden and T. J. Suffridge, Biholomorphic maps in Hilbert space have a fixed point, Pacific J. Math. 38 (1971), 419-422.

76. M. Hervé, Analytic continuation on Banach spaces, Several Complex Variables II, Lecture Notes in Math., vol. 185, Springer-Verlag, Berlin, 1971, pp. 63-75.

77. - Analytic and plusisubharmonic functions in finite and infinite dimensional spaces, Lecture Notes in Math., vol. 198, Springer-Verlag, Berlin, 1971.

78. A. Hirschowitz, Sur le non-plongement des variétés analytiques banachiques réelles, C. R. Acad. Sci. Paris Sér. A-B 269 (1969), A844-A846. MR 40 \#6591.

79. - Remarques sur les ouverts d'holomorphie d'un produit dénombrable de droites, Ann. Inst. Fourier (Grenoble) 19 (1969), 219-229. MR 40 \# 5892.

80. _ Sur les suites de fonctions analytiques, Ann. Inst. Fourier (Grenoble) 20 (1970), fasc. 2, 403-413. MR 44 \# 3104.

81. - Prolongement analytique en dimension infinie, C. R. Acad. Sci. Paris Sér. A-B 270 (1970), A1736-A1737. MR 42 \#3307.

82. - Diverses notions d'ouverts d'analyticité en dimension infinie, Séminaire Pierre

Lelong 1970, Lecture Notes in Math., vol. 205, Springer-Verlag, Berlin, 1971, pp. 11-20.

83. __, Bornologie des espaces de fonctions analytiques en dimension infinie, Séminaire

Pierre Lelong 1970, Lecture Notes in Math., vol. 205, Springer-Verlag, 1971, pp. 21-33.

84. _ Prolongement analytique en dimension infinie, Ann. Inst. Fourier (Grenoble) 22 (1972), 255-292.

85. — Sur un théorème de M. A. Zorn, Arch. Math. 23 (1972), 77-79.

86. - Convexité abstraite et espaces normés, Séminaire Pierre Lelong 1970/71,

Lecture Notes in Math., vol. 275, Springer-Verlag, Berlin, 1972, pp. 1-13.

87. - See Dineen, S. [44].

88. H. Hogbe-Nlend, Deux remarques sur les applications analytiques en dimensions infinie, An. Acad. Brasil. Ci. (to appear).

89. - Applications analytiques entre espaces et algèbres bornologiques, Colloq. sur les Fonctions Analytiques de Plusieurs Variables Complexes (Paris, 1972), Centre National de la Recherche Scientifique, Paris (to appear).

90. - Sur la métrisabilité des espaces de fonctions holomorphes en dimension infinie, Bull. Sci. Math. (to appear).

91. C. O. Kiselman, On entire functions of exponential type and indicators of analytic functionals, Acta Math. 117 (1967), 1-35. MR 35 \# 1825.

92. - Plurisubharmonic functions in vector spaces, Report \#39, Uppsala University Department of Mathematics, Sweden, 1972.

93. ㄴ. See Gruman, L. [64].

94. D. Lazet, Sur la différentiabilité des applications analytiques en dimension infinie, C. R. Acad. Sci. Paris Sér. A-B 273 (1971), A155-A157. MR 44 \#800.

95. ㄴ. Applications analytiques dans les espaces bornologiques, Université de Bordeaux, France, 1972.

96. - See Colombeau, J.-F. [37].

97. P. Lelong, Fonctionnelles analytiques et fonctions entières, Séminaire de Mathématiques Supérieures, Université de Montréal, Canada, 1967.

98. - Fonctions plurisousharmoniques dans les espaces vectoriels topologiques, Séminaire Pierre Lelong 1967/68, Lecture Notes in Math., vol. 71, Springer-Verlag, Berlin, 1968, pp. $167-189$.

99. - Fonctions plurisousharmoniques et ensembles polaires dans les espaces vectoriels topologiques, C. R. Acad. Sci. Paris Sér. A-B 267 (1968), A916-A918. MR 39 \# 7412.

100. , Fonctions et applications de type exponentiel dans les espaces vectoriels topologiques, C. R. Acad. Sci. Paris Sér. A-B 269 (1969), A420-A422. MR 40 \# 3293.

101. - Fonctions plurisousharmoniques et ensembles polaires sur une algèbre de fonctions holomorphes, Séminaire Pierre Lelong 1969, Lecture Notes in Math., vol. 116, Springer-Verlag, Berlin, 1970, pp. 1-20.

102. - Recent results on analytic mappings and plurisubharmonic functions in topological linear spaces, Several Complex Variables II, Lecture Notes in Math., vol. 185, Springer-Verlag, Berlin, 1971, pp. 97-124. 
103. Théorème de Banach-Steinhaus pour les polynômes: applications analytiques entières d'espaces vectoriels, Séminaire Pierre Lelong 1970, Lecture Notes in Math., vol. 205, Springer-Verlag, Berlin, 1971, pp. 87-112.

104. - Sur les fonctions plurisousharmoniques dans les espaces vectoriels topologiques et une extension du theorème de Banach-Steinhaus aux familles d'applications polynoliales, Troisieme Colloque sur l'Analyse Fonctionelle (Liège, 1970), Centre Belge de Recherches Mathématiques, Vander, Belgium, 1971, pp. 21-45.

105. - Fonctions plurisousharmoniques dans les espaces vectoriels topologiques, Colloq. sur les Fonctions Analytiques de Plusieurs Variables Complexes (Paris, 1972), Centre National de la Recherche Scientifique, Paris (to appear).

106. M. C. Matos, Holomorphic mappings and domains of holomorphy, Monografias do Centro Brasileiro de Pesquisas Físicas, no. 27, Rio de Janeiro, Brasil, 1970.

107. - Sur les applications holomorphes définies dans les espaces vectoriels topologiques de Baire, C. R. Acad. Sci. Paris Sér. A-B 271 (1970), A599. MR 42 \# 5045.

108. - Sur l'enveloppe d'holomorphie des domaines de Riemann sur un produit dénombrable de droites, C. R. Acad. Sci. Paris Sér. A-B 271 (1970), A727-A728. MR 42 \#3554.

109. - Sur les ouverts de $\tau$-holomorphie dans les espaces de Banach séparables, C. R. Acad. Sci. Paris Sér. A-B 271 (1970), A1165-A1166. MR 42 \#4766.

110. - Sur le théorème d'approximation et d'existence de Malgrange-Gupta, C. R. Acad. Sci. Paris Sér. A-B 271 (1970), A1258-A1259. MR 44 \#3105.

111. - The envelope of holomorphy of Riemann domains over a countable product of complex planes, Trans, Amer. Math. Soc. 167 (1972), 379-387.

112. , Domains of $\tau$-holomorphy in a separable Banach space, Math. Ann. 195 (1972), 273-278.

113. Mat. Ital. (4)6 (1972), 67-70.

114. in a locally convex space, J. Reine Angew. Math. (to appear).

115. P. Mazet, Le "nullstellensatz" pour un germe analytique banachique, C. R. Acad. Sci. Paris Sér. A-B 269 (1969), A235-A237. MR 42 \# 2037.

116. —_ Nullstellensatz en géométrie analytique banachique, Séminaire Pierre Lelong 1970, Lecture Notes in Math., vol. 205, Springer-Verlag, Berlin, 1971, pp. 71-86.

117. - Le théorème des zeros dans le cas banachique, Espaces Analytiques (Séminaires Bucharest, 1969), Editura Acad. R.S.R., Bucharest, 1971, pp. 137-143. MR 43 \#6461.

118. - Propriétés de $\mathcal{O}(E)$ vis-à-vis des decompositions primaires, Séminaire Pierre

Lelong 1970/71, Lecture Notes in Math., vol. 275, Springer-Verlag, Berlin, 1972, pp. 71-81.

119. - Généralisation de la notion d'anneau noethérien. Applications à la géométrie de dimension infinie, Colloq. Fonctions Analytiques de Plusieurs Variables Complexes (Paris, 1972), Centre National de la Recherche Scientifique, Paris (to appear).

120. L. Nachbin, Lectures on the theory of distributions, University of Rochester, Rochester, N.Y., 1963; reproduced in Textos de Matemática, no. 15, Instituto de Física e Matemática, Universidade do Recife, Recife, 1964. MR 35 \#4722.

121. - On the topology of the space of all holomorphic functions on a given open set,

Nederl. Akad. Wetensch. Proc. Ser. A 70=Indag. Math. 29 (1967), 366-368. MR 35 \# 5910.

122. - On spaces of holomorphic functions of a given type, Functional Analysis

(Irvine, 1966), Academic Press, New York, 1967, pp. 55-70. MR 39 \# 3298.

123. - Topology on spaces of holomorphic mappings, Ergebnisse der Mathematik und ihrer Grenzgebiete, Heft 47, Springer-Verlag, Berlin, 1969. MR 40 \# 7787.

124. Convolution operators in spaces of nuclearly entire functions on a Banach space, Functional Analysis and Related Fields (Chicago, 1968), Springer-Verlag, Berlin, 1970, pp. 167-171.

125. on Nuclear Spaces and Ideals in Operator Algebras (Warsaw, 1969), Studia Math. 38 (1970), 407-412. MR 43 \#3787.

126. ㄱ. Concerning spaces of holomorphic mappings, Rutgers University, New Brunswick, N.J., 1970.

127. - Sur les espaces vectoriels topologiques d'applications continues, C. R. Acad. Sci. Paris Sér. A-B 271 (1970), A596-A598. MR 42 \#6593.

128. - Uniformité d' holomorphie et type exponentiel, Séminaire Pierre Lelong 1970, Lecture Notes in Math., vol. 205, Springer-Verlag, Berlin, 1971, pp. 216-224.

129. — Convoluções em funções inteiras nucleares, Atas da Segunda Quinzena de 
Análise Funcional e Equações Diferenciais Parciais (São José dos Campos, 1969), Sociedade Brasileira de Matemática, Brasil, 1972, pp. 1-51.

130. - Sur quelques aspects récents de l'holomorphie en dimension infinie, Séminaire Goulaouic-Schwartz, École Polytechnique, Paris, 1971/72, 18 (1-9).

131. - Limites et perturbation des applications holomorphes, Colloq. sur les Fonctions Analytiques de Plusieurs Variables Complexes (Paris, 1972), Centre National de la Recherche Scientifique, Paris (to appear).

132. . See Barroso, J. A. [10], Dineen, S. [52].

133. $\mathrm{Ph}$. Noverraz, Un théorème de Hartogs et théorèmes de prolongement dans les espaces vectoriels topologiques complexes, C. R. Acad. Sci. Paris Sér. A-B 266 (1968), A806-A808. MR 37 \#5423.

134. - Fonctions plurisousharmoniques et analytiques dans les espaces vectoriels topologiques complexes, Ann. Inst. Fourier (Grenoble) 19 (1970), fasc. 2, 419-493. MR 42 \#537.

135. - Fonctions analytiques et théorèmes de prolongement dans les espaces vectoriels topologiques complexes, Séminaire Pierre Lelong 1969, Lecture Notes in Math., vol. 116, Springer-Verlag, Berlin, 1970, pp. 21-29.

136. - Sur la convexité fonctionnelle dans les espaces de Banach à base, $\mathrm{C} . \mathrm{R}$. Acad. Sci. Paris Sér. A-B 272 (1971), A1564-A1566. MR 43 \# 7659.

137. - Convexité fonctionnelle dans les espaces de Banach à base, Séminaire Pierre Lelong 1970/71, Lecture Notes in Math., vol. 275, Springer-Verlag, Berlin, 1972, pp. $166-176$.

138. - Sur la convexité fonctionnelle en dimension infinie, C. R. Acad. Sci. Paris Sér. A-B 274 (1972), A313-A315.

139. - Pseudo-convexité, convexité polynomials et domaines d'holomorphie en dimension infinie, Notas de Matemática, no. 48, North-Holland, Amsterdam, 1973.

140. - Notions de domaines d'holomorphie dans les espaces de Banach complexes, Atas do Oitavo Colóquio Brasileiro de Matemática (Poços de Caldas, 1971), Instituto de Matemática Pura e Aplicada, Rio de Janeiro, Brasil (to appear).

141. - Sur le théorème de Cartan-Thullen-Oka en dimension infinie, An. Acad. Brasil. Ci. (to appear).

142. - Pseudo-convexité et completion holomorpheen dimension infinie, Colloq. sur les Fonctions Analytiques de Plusieurs Variables Complexes (Paris, 1972), Centre National de la Recherche Scientifique, Paris (to appear).

143. - - Sur la pseudo-convexité et la convexité polynomiale en dimension infinie, Ann. Inst. Fourier (Grenoble) (to appear).

144. D. Pisanelli, Sui funzionali transcendenti interi dello spazio LN*, Boll. Un. Mat. Ital. (3) 19 (1964), 106-109. MR 30 \# 2314.

145. _ Sull estensione del teorema di Montel, Atti Accad. Naz. Lincei Rend. Cl.

Sci. Fis. Mat. Natur. (8) 46 (1969), 137-139. MR 40 \#3262.

146. - Sur les applications analytiques en dimension infinie, C. R. Acad. Sci. Paris Sér. A-B 274 (1972), A760-A762.

147. ㄴ Sur la $(L F)$-analyticité, Colloq. Analyse (Rio de Janeiro, 1972), Hermann, Paris (to appear).

148. ㄴ. Applications analytiques en dimension infinie, Bull. Sci. Math. (to appear).

149. J.-P. Ramis, Sous-ensembles analytiques d'une variété banachique complexe, Ergebnisse der Mathematik und ihrer Grenzgebiete, Heft 53, Springer-Verlag, Berlin, 1970.

150. C. E. Rickart, Analytic phenomena in general function algebras, Pacific J. Math. 18 (1966), 361-377. MR 33 \#6438.

151. Holomorphic convexity for general function algebras, Canad. J. Math. 20 (1968), 272-290. MR 37 \#3362.

152. (1969), 581-597. MR 40 \# 7819.

153. - Extension of results from several complex variables to general function algebras, Lectures in Modern Analysis and Applications. I, Lecture Notes in Math., vol. 103, Springer-Verlag, Berlin, 1969, pp. 44-59. MR 39 \# 7437.

154. - Plurisubharmonic functions and convexity properties for general function algebras, Trans. Amer. Math. Soc. 169 (1972), 1-24.

155. - A function algebra approach to infinite dimensional holomorphy, Colloq. Analyse (Rio de Janeiro, 1972), Hermann, Paris (to appear).

156. G. Ruget, $\grave{A}$ propos des cycles analytiques de dimension infinie, Invent. Math. 8 (1969), 267-312. MR 42 \#7945.

157. M. Schottenloher, Über analytische Fortsetzung in Banachräumen, Math. Ann. 199 
(1972), 313-336.

158. - The envelope of holomorphy as a functor, Colloq. sur les Fonctions Analytiques de Plusieurs Variables Complexes (Paris, 1972), Centre National de la Recherche Scientifique, Paris (to appear).

159. - Bounding sets in Banach spaces and regular classes of analytic functions, Sympos. Analysis (Recife, 1972), Lecture Notes in Math., Springer-Verlag, Berlin (to appear). 160. $-\varepsilon$-product and continuation of analytic mappings, Colloq. Analyse (Rio de Janeiro, 1972), Hermann, Paris (to appear).

161. - Analytic continuation in locally convex Hausdorff spaces, An. Acad. Brasil.

Ci. (to appear).

162. J. Siciak, A generalization of a polynomial lemma of Leja, Ann. Polon. Math. 24 (1972), 149-156.

163. - A polynomial lemma and analytic mappings in topological vector spaces,

Séminaire Pierre Lelong 1970/71, Lecture Notes in Math., vol. 275, Springer-Verlag, Berlin, 1972, pp. 131-142.

164. . See Bochnak, J. [13], [14], [15], [16].

165. J. O. Stevenson, Holomorphy of composition, Thesis, University of Rochester, Rochester, N.Y., 1972.

166. T. J. Suffridge. See Hayden, T. L. [75].

167. A. E. Taylor, Historical notes on analyticity as a concept in functional analysis, Problems in Analysis, Princeton Math. Series, vol. 31, Princeton Univ. Press, Princeton, N.J., 1970, pp. 325-343.

168. - Notes on the history of the uses of analyticity in operator theory, Amer. Math. Monthly 78 (1971), 331-342. MR 43 \#5331.

169. L. Waelbroeck, Topological vector spaces and algebras, Lecture Notes in Math., vol. 230, Springer-Verlag, Berlin, 1971

170. N. R. Wallach. See Greenfield, S. J. [62], [63].

171. H. E. Warren, A Riemann mapping theorem for $C(X)$, Proc. Amer. Math. Soc. 28 (1971), 147-154. MR 43 \# 5300.

Current address: Avenida Vieira Souto 144, apto. 101, Rio de Janeiro, Guanabara, ZC-95, Brasil. 